\title{
The theoretical basis for the prediction of early strength plasticized cement stone
}

\author{
Andrei Serenko ${ }^{1, *}$ and Aleksandr Golovko \\ ${ }^{1}$ Far Eastern State Transport University, ul. Serysheva, 47, Khabarovsk, 680000, Russia
}

\begin{abstract}
Theoretical analyses and experimental data to predict durability of plasticized cement systems at an early age. Proposed to use to assess the compatibility and selection of cement and chemical additives on the early stage hydration of power rate dependence of M. Balhina. Methodology and mathematical dependences for determining the power indicator at an early age.
\end{abstract}

\section{Introduction}

Water-reducing effect of superplasticizers, accompanied by decreasing water-cement ratio, leads to significant difficulties in predicting the strength of cement systems at an early age. The following main causes of this processcan be formulated:

1) Dependence of the strength of cement systems from porosity, and, consequently, of the value of water-cement ratio, has a complicated nonlinear character. Formulas of M.Yu. Balshin, K.K. Schillerand E. Ryzhkevich [1] are, respectively, exponential, logarithmic, and exponential.

2) By research of J. Jambor, V.V. Babkov, V.N. Mokhov, S.M. Kapitonov, P.G. Komokhov [2] it is proved that in contrast to single-phase porous materials, durability of cement systems cannot definitely be characterized through the total or capillary porosity, because mentioned parameters are not able to fully consider the impact of the structure of cement stone in its strength. It can be expected that this dependence will be sustainable only to cement systems with constant mineralogical and chemical composition with relatively close values of water-cement ratio and the same conditions of hardening, as well as when using only one type chemical additives.

3) In the fundamental works of G.I. Gorchakov, O.V. Kuntsevich, A.E. Sheykin etc. it is evidentially proved that the magnitude of the capillary porosity of the cement stone is determined by the water-cement ratio and the cement's hydration degree. The difficulty of determining the rate of hydration at the early stage of hardening introduces additional uncertainty in the determination of dependence between the strength and structural characteristics of cement stone.

4) Since when using the superplasticizers in parallel with a reduction of water-cement ratio there is a retardation of hydration processes, during predicting the strength of cement at an early age it is necessary to consider the blocking effect of the additives on the hardening process.

\footnotetext{
*Corresponding author: dek_its@festu.khv.ru
} 


\section{Theory section}

Mentioned preconditions must be considered in prediction of early strength of cement systems while reducing water-cement ratio through the use of plasticizing additives.

To establish the dependence of the early strength from the structural parameters of cement stone when reducing water-cement ratio by us, the power dependence of M.Yu. Balshin in a modified form was taken as a base:

$$
R=R_{g c} \cdot V^{S}
$$

where $R$ - the strength of cement stone;

$R_{g c}$ - the strength while crystal-hydrate strap's compression, corresponding to the strength of cement stone at zero capillary porosity;

$V=1-P_{\kappa}$ - the relative volumetric concentration of solid phase (without the capillary pores);

$P_{\kappa}$ - the value of the capillary porosity of a cement stone;

$S$ - the power indicator reflecting the intensive nature of strength's increase with a growth of volume concentration of solid phase.

$\mathrm{M} . Y u$. Balshin's selection of dependence through the bond of the cement stone'sstrength with a capillary porosity is based on the fact that under these settings F. Locher obtained the highest correlation for cement systems that harden at normal humidity conditions. In this case, the crystal-hydrate growth with its inherent gel pores are treated as a homogeneous solid phase. The influence of conditionally closed pores on the change in strength while decreasing water-cement ratio in the same cement system can be neglected, if the reduction of water-cement ratiois carried out in the cement systems of the same composition, because in this case, the content of entrapped air remains approximately the same.

If to define the strength of the unplasticized cement stone as $R^{n}$, and plasticized cement stone with a reduced water-cement ratio as $R^{p l}$, then the change in strength can be expressed through the ratio of the resistances in accordance with (1):

$$
\frac{R^{p l}}{R^{n}}=\frac{R_{g c} \cdot\left(V_{p l}\right)^{S}}{R_{g c} \cdot\left(V_{n}\right)^{S}}=\left(\frac{V_{p l}}{V_{n}}\right)^{S},
$$

where $V_{n}$ and $V_{p l}$ - the relative volumetric concentration of the solid phase of unplasticized and plasticized cement stone;

$S$ - the power indicator.

Thus, the problem of determining the influence of water-reducing action of plasticized additives on the stability of cement stone comes up to finding the values of the power indicator and the establishment of indicators of the cement stone structure, determining a value of the relative volumetric concentration of solid phase in a controlled age. From the theoretical positions, while the process of the hydration the volumetric concentration of solid phase and its structure will change, that can not affect the value of the power indicator $S$. Due to the given above limitations, we should expect that the values of the power index will be different for each cement system and can differ within a single system in relation to the reference age and the additives used. (2):

The value of the power indicator can be found by taking the prelogarithmic equation 


$$
S=\frac{\lg \left(R^{p l} / R^{n}\right)}{\lg \left(V_{p l} / V_{n}\right)}
$$

Finding the dependence of the relative volumetric concentration of the solid phase on the parameters of cement stone is doable under the following assumptions: the volume of cement paste does not change during hydration of cement; the increment of the volume of the solid phase in proportion to the degree of hydration of cement; the cement gel with the normal structure in which the pore size does not exceed $40 \cdot 10^{-10} \mathrm{~m}$, is considered as one solid phase; the pores of the cement gel when it is aging in excess of $40 \cdot 10^{-10} \mathrm{~m}$ aresubmicromolar.

Under these assumptions, the derivation of the equation comes to the following. The volume of the solid phase when the degree of hydration is $\alpha$ :

$$
v=v_{c}+\Delta v,
$$

where $v_{c}$ - the volume of cement;

$\Delta v$ - the increment of the volume of the solid phase when the degree of hydration is $\alpha$.

The increment of the volume of the solid phase when the degree of hydration is $\alpha$ will be determined by the formula of O. V. Kuntsevich [3]:

$$
\Delta v=\alpha \cdot\left(K_{g} \cdot N-1\right) \cdot C / \rho_{c},
$$

where $K_{g}=0,72 \div 1,0-$ the speed of ageing of the gel;

$N$ - thegel-cement ratio equal to the ratio of volume of gel to volume of cement;

$C$ - the weight of the cement;

$\rho_{c}$ - the density of the cement.

Inserting (5) into (4) and expressing the volume of cement through its weight and density, we get:

$$
v=\left(1+\alpha \cdot\left(K_{g} \cdot N-1\right)\right) \cdot C / \rho_{c}
$$

Then the relative volume of the solid phase when the degree of hydration is $\alpha$ :

$$
V=\frac{v}{v_{c t}}=\frac{v}{v_{w}+v_{c}}
$$

where $v_{u m}$ - the volume of cement paste;

$v_{w}$ - the volume of water;

$v_{c}$ - the volume of cement.

Expressing the volume of water and cement through their weight and density, and bringing it to a common denominator, we get:

$$
v_{w}+v_{c}=C \cdot\left(W / C+\rho_{w} / \rho_{c}\right) / \rho_{w},
$$

where $\rho_{w}$ and $\rho_{c}$ - the density of water and cement.

Inserting (8) and (6) into (7) and performing the reduction, we get: 


$$
V=\frac{\rho_{w} \cdot\left(1+\alpha \cdot\left(K_{g} \cdot N-1\right)\right)}{\rho_{c} \cdot\left(W / C+\rho_{w} / \rho_{c}\right.}
$$

Denoting the ratio of the densities of water and cement through $\vartheta=\rho_{w} / \rho_{c}$, we will come to a following statement:

$$
V=\frac{\vartheta \cdot\left(1+\alpha \cdot\left(K_{g} \cdot N-1\right)\right)}{W / C+\vartheta}
$$

or, dividing a numerator and a denominator by $\vartheta$ :

$$
V=\frac{1+\alpha \cdot\left(K_{g} \cdot N-1\right)}{1+\frac{W / C}{\vartheta}}
$$

Therefore, the main structural parameters determining the relative volumetric concentration of solid phase with pores of the gel in it are water-cement ratio and degree of hydration of cement. Indirect confirmation of the adequacy of the obtained expression can be obtained by considering the relative volume of cement in cement paste:

$$
V_{c}=\frac{v_{c}}{v_{c t}}=\frac{v_{c}}{v_{w}+v_{c}}
$$

where $V_{c}$ - the relative volume concentration of cement in cement paste;

$v_{c t}, v_{w}, v_{c}$ - the volumes of cement paste, water and cement.

Expressing the volume of water and cement through their weight and density, we get:

$$
V_{c}=\frac{1}{1+\frac{W \cdot \rho_{c}}{C \cdot \rho_{w}}}
$$

Denoting the ratio of the densities of water and cement through $\vartheta=\rho_{w} / \rho_{c}$, we will come to a following statement:

$$
V_{c}=\frac{1}{1+\frac{W / C}{\vartheta}}
$$

The relative volume of the solid phase of the cement stone with the degree of hydration of cement $\alpha=0$ must be equal to the relative volume of cement. Comparing expressions (14) and (11) if $\alpha=0$, we will be convinced of their identity, which indirectly confirms the validity of the proposed expressions for calculating the relative volume concentration of the solid phase of the cement stone. Power indicator S,connecting structure parameters of plasticized and unplasticized cement paste with size of its strength, we find, substituting the statement (11) into the formula (3):

$$
S=\frac{\lg \frac{R^{p l}}{R^{n}}}{\lg \frac{\left(1+\alpha_{p l} \cdot\left(K_{g} \cdot N-1\right)\right) \cdot\left(W / C_{n}+\vartheta\right)}{\left(1+\alpha_{n} \cdot\left(K_{g} \cdot N-1\right)\right) \cdot\left(W / C_{p l}+\vartheta\right)}}
$$


If to take at early age the rate of aging of the gel $K_{g}$ per unit, the density of the cement 3,125 , and the density of water is 1.0 , that provides the value $\vartheta=0,32$, and take into account data of Powers on the amount of gel-cement relation, constituting $N=2,2$ [4], we get the following dependence for determining the value of the power indicator $S$ :

$$
S=\frac{\lg \left(R^{p l} / R^{n}\right)}{\lg \frac{\left(1+1,2 \cdot \alpha_{p l}\right) \cdot\left(W / C_{n}+0,32\right)}{\left(1+1,2 \cdot \alpha_{n}\right) \cdot\left(W / C_{p l}+0,32\right)}}
$$

The resulted mathematical statement can be used to predict the influence of waterreducing action of plasticizers on the strength of cement stone at an early age taking into account the mentioned above restrictions. We can assume that the power indicator $S$ can be calculated for cements of the same type in the same age with the use of additives of the same type.

A significant difficulty is the determination of the degree of hydration at early age of hardening, because the existing methods are labour-consuming and often require time spending and special equipment, the possibility of continued hydration during the test is not excluded, that can lead to significant deviations of the results obtained from the true values.

\section{Experimental section}

To analyze the effect of hardening time on the exponent of power dependence of M.Yu. Balshin and reliability of the results at different ages, the following series of experiments were made. On the basis of the Belgorod cement PC500 DO the samples of cement stone were manufactured from equal-plastic cement paste, corresponding to normal density. Superplasticizer C-3 was used as a plasticizer at a dose of from 0.5 to $0.9 \%$ with a step of $0.2 \%$. The samples were hardened in normal humidity conditions and in the age of 12 hours, 1,3 and 28 days were tested for the strength while compression. Parallel to the strength tests determining the extent of hydration heat was carried out according to the amount of bound water, based on the position that at full hydration the amount of chemically bound water is $22 \%$. Based on the data obtained by the formula (16) the index of power dependence was calculated and its mean value $\left(S_{c p}\right)$ was determined for the controlled age. The calculated values of the strength of cement stone on the basis of the obtained average value of the index of power dependence of M.Yu. Balshin $\left(S_{c p}\right)$ was determined by deriving the formula (16) expression (17):

$$
R^{p l}=R^{n} \cdot\left(\frac{\left(1+1,2 \cdot \alpha_{p l}\right) \cdot\left(W / C_{n}+0,32\right)}{\left(1+1,2 \cdot \alpha_{n}\right) \cdot\left(W / C_{p l}+0,32\right)}\right)^{S_{c p}}
$$

The comparison of actual and calculated values of the strength of cement stone allowed to determine the coefficient of its correlation for each period of hardening. The variability of the power indicator $\mathrm{S}$ depending on the duration of the hardening cement stone was confirmed. With increasing time of hardening the average value of the index of power dependence of M.Yu. Balshinplasticized cement stone increases (fig. 1) 


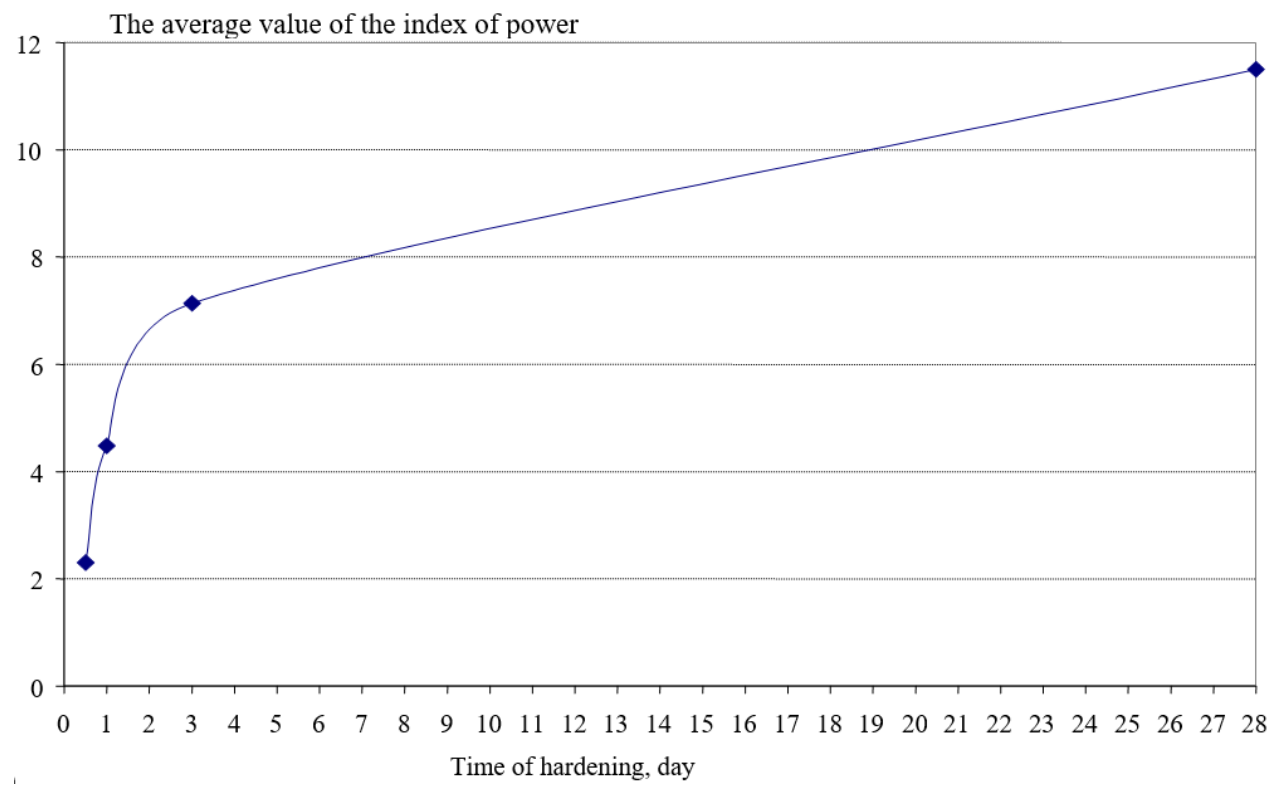

Fig. 1. Influence of duration of hardening on the average value of the power indicator.

Gradual increase of values of the power indicator of the dependence of M.Yu. Balshin from daily to 28-days age is associated with a sequential accumulation of fine hydration products. A significant reduction of power indicator at the age less than 1 day, tells about significant changes to the structure and confirms the hypothesis about the change of ratio of pores of cement stone in size [5].

The performed studies allowed us to estimate the effect of age of cement stone on the validity of the results obtained by the proposed method of predicting of strength (fig. 2 and $3)$.

\section{Coefficient of correlation}

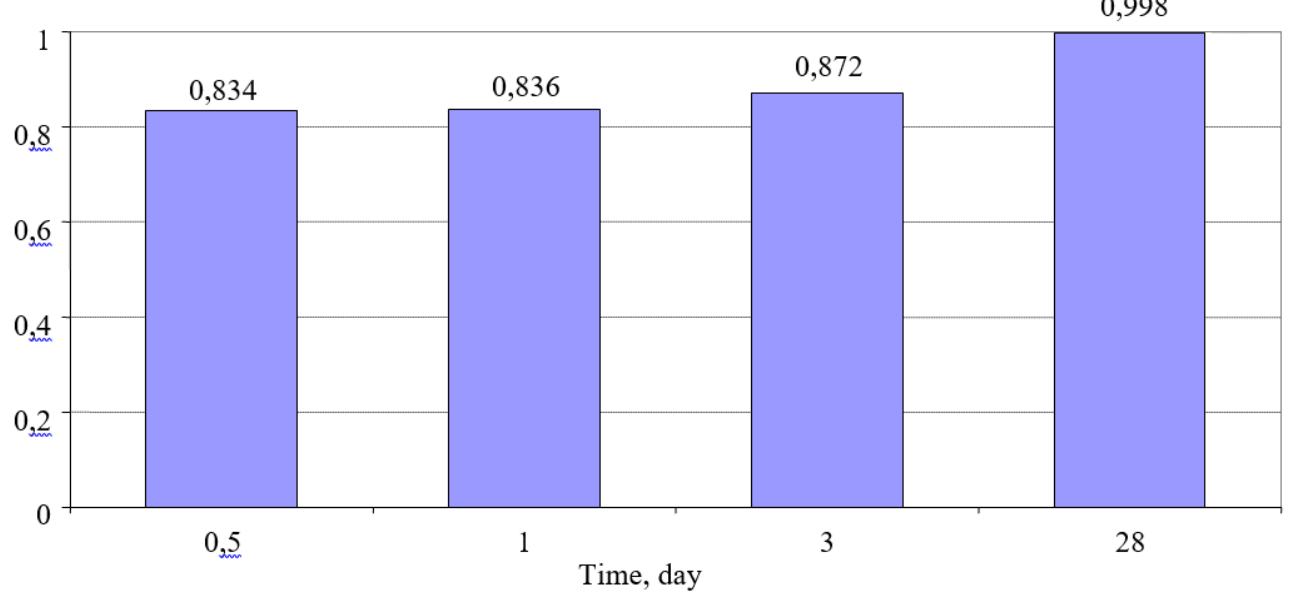

Fig. 2. Influence of hardening time on the coefficient of correlation of calculated and actual values of the strength of cement stone. 


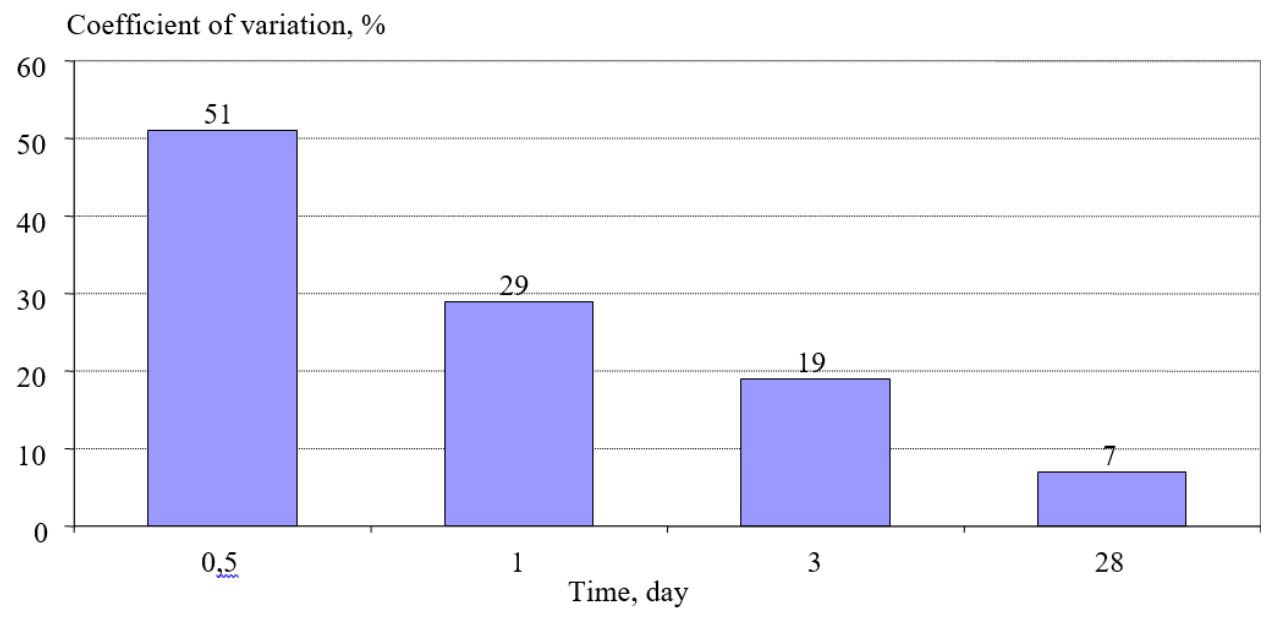

Fig. 3. Influence of hardening time on the coefficient of variation of the power dependence of M.Yu. Balshin.

High correlation between actual and calculated values of strength obtained on the base of average values of the index of power dependence of M.Yu. Balshin, observed at the age of 28 days and has a magnitude more than 0.99 . At the early age of hardening the accuracy of determining the power index decreases, which follows from the data on the effect of age of cement stone on the coefficient of variation of power law index (Fig. 3), which consistently reduces from 51 to $7 \%$.

The decrease in the determination accuracy of the index of power dependence of M.Yu. Balshinwith a reduction in age was attributable to higher requirements on the accuracy of finding the degree of hydration of cement in connection with decreasing absolute values and large scatter of results when you change the dosage of C-3 due to the action of the blocking effect and changes in the ratio of pores of cement stone in size.

\section{Conclusions}

Formulas (16) and (17) allow to draw some private conclusions about the ratio of the strengths of plasticized and unplasticized cement stone. Considering water-reducing action of plasticizing additives $W_{d}$,representing the ratio of the water cement relationship of plasticized and unplasticized cement, equation (17) transforms into the statement:

$$
R^{p l}=R^{n} \cdot\left(\frac{\left(1+1,2 \cdot \alpha_{p l}\right) \cdot\left(W / C_{n}+0,32\right)}{\left(1+1,2 \cdot \alpha_{n}\right) \cdot\left(W_{d} \cdot W / C_{n}+0,32\right)}\right)^{S_{c p}}
$$

And at the same degree of hydration of plasticized and unplasticized cement stone:

$$
R^{p l}=R^{n} \cdot\left(\frac{\left(W / C_{n}+0,32\right)}{\left(W_{d} \cdot W / C_{n}+0,32\right)}\right)^{S_{c p}}
$$

The index of power dependence of M.Yu. Balshindetermined from the statement (16) can take both positive and negative values, associated with the ratio of the strengths of plasticized and unplasticized cement stone. In case of equality of strengths of plasticized and unplasticized cement stone, which speaks of the mutual compensation of changes in strength due to the water reducing and blocking additives, power indicator takes the value 
of zero. With the prevalence of blocking activity of additive over the water reducing effect the value of the power index takes a negative value. Therefore, the index of power dependence of M.Yu. Balshinserve as a criterion to assess compatibility and the choice of portland-cement and chemical additives. For fast-hardening concretes, the value of the index of power dependence of M.Yu. Balshinat an early age must be more than zero. The higher its absolute value, ceteris paribus, the better the compatibility of cement and chemical additives from the point of view of obtaining a fast-hardening concretes.

\section{References}

1. R.W. Rice, Microstructure Dependence of Mechanical Behavior of Ceramics (Bibliogr., New York, 1977)

2. V.V. Babkov, V.N. Mokhov, S.M. Kapitonov, P.G. Komokhov, The structure formation and destruction of cement concrete (SUE Ufa polygraphic Plant, Ufa, 2002)

3. V.O. Kuntsevich, Concrete of high frost-resistance for buildings constructions in the Far North (Stroyizdat, Moscow, 1983)

4. T.S. Powers, Physical structure of Portland-cement paste. The chemistry of cements (Stroyizdat, Moscow, 1969)

5. T.M. Petrova, A.F. Serenko, Bulletin of civil engineers 3(16), 76-80 (2008) 